\title{
Examining Lung Cancer Screening Behaviors in the Primary Care Setting: A Mixed Methods Approach
}

\author{
Alvie Ahsan ${ }^{1}$, Eva Zimmerman ${ }^{1}$, Elisa Marie Rodriguez ${ }^{2}$, Christy Widman $^{2}$, Deborah Oates Erwin ${ }^{2}$, \\ Frances Georgette Saad-Harfouche ${ }^{2}$, Martin Christopher Mahoney ${ }^{2,3}$, * \\ ${ }^{1}$ Jacobs School of Medicine and Biomedical Sciences, State University at New York at Buffalo, Buffalo, USA \\ ${ }^{2}$ Department of Cancer Prevention \& Control, Roswell Park Comprehensive Cancer Center, Buffalo, USA \\ ${ }^{3}$ Department of Internal Medicine, Roswell Park Comprehensive Cancer Center, Buffalo, USA
}

Email address:

Martin.Mahoney@RoswellPark.org (M. C. Mahoney)

*Corresponding author

\section{To cite this article:}

Alvie Ahsan, Eva Zimmerman, Elisa Marie Rodriguez, Christy Widman, Deborah Oates Erwin, Frances Georgette Saad-Harfouche, Martin Christopher Mahoney. Examining Lung Cancer Screening Behaviors in the Primary Care Setting: A Mixed Methods Approach. Journal of Cancer Treatment and Research. Vol. 7, No. 1, 2019, pp. 1-8. doi: 10.11648/j.jctr.20190701.11

Received: January 15, 2019; Accepted: February 16, 2019; Published: March 11, 2019

\begin{abstract}
While the National Lung Screening Trials (NLST) demonstrated the efficacy of low dose chest computed tomography (LDCT) for lung cancer early detection, utilization of LDCT remains suboptimal. The purpose of this formative study was to understand attitudes and beliefs among primary care clinicians regarding LDCT lung cancer screening as well as to assess gaps in knowledge to identify opportunities for reinforcing personalized lung cancer screening that is accessible and evidence-based. A 20-item closed and open-ended interview was conducted with a targeted group of primary care clinicians (38 respondents; 33 physicians and $5 \mathrm{NPs} / \mathrm{PAs}$ ). Quantitative data were analyzed using descriptive statistics while qualitative data was analyzed thematically. Although $50.0 \%$ of clinicians characterized LDCT as "very effective", only $47.4 \%$ of clinicians reported that they frequently or often recommend LDCT as a lung cancer screening tool. Respondents were generally unconcerned with the high rate of false positive test results. The majority of clinicians were referring patients for LDCT based on smoking history, however other factors were also considered (e.g., health status, sex, family history, past medical history, and occupational exposures.) The majority of respondents were knowledgeable about the use of LDCT as a lung screening tool but were unsure about its effectiveness for lung cancer early detection. Some clinicians are recommending patients for LDCT based on factors which are inconsistent with evidence-based guidelines.
\end{abstract}

Keywords: Lung Cancer Screening, Early Detection, Clinician Education, Primary Care, Provider Perception

\section{Introduction}

Lung cancer is currently the leading cause of cancerassociated death, and is the third most common cancer diagnosis following prostate and breast cancer in the United States [1]. The vast majority of lung cancer cases are associated with tobacco use [2]. The five-year lung cancer survival rate $(17.8 \%)$ is relatively low especially when compared to breast and prostate cancers, which both have five-year survival rates greater than $90 \%$. While the survival rate increases when lung cancer is detected at an early stage, only $15 \%$ of lung cancers are diagnosed at an early, localized stage. More than one-half of those who are diagnosed with lung cancer die within a year of their diagnosis due to advanced stage disease [3].

In 2013, the United States Preventative Services Task Force (USPSTF) issued a Grade B recommendation for annual lung cancer screening among asymptomatic adults ages 55 to 80 years who have a 30 pack-year smoking history and who currently smoke or have quit smoking within the last 15 years [4]. Results from the National Lung Screening Trial (NLST) which compared lung cancer mortality among participants screened with low-dose helical computed tomography (LDCT) to participants who were screened with chest radiographs (CXR) demonstrated a $20.0 \%$ decreased mortality from lung cancer in those who were screened with 
LDCT compared with those who were screened with CXR [5].

While a handful of studies have explored physician attitudes and experiences with LDCT screening [6-9], few have made use of a qualitative design. Kanodra et al., (2016) used a qualitative design to explore perceptions of lung cancer screening among primary care physicians (PCPs). This study, which was conducted at a single VA facility and accrued 13 PCPs, noted that nearly $60 \%$ were aware of USPSTF guidelines, but were unable to recall eligibility criteria and expressed variable interest in completing lung cancer screening [10]. Another qualitative study explored shared decision-making on the topic of lung cancer screening among 36 clinicians from three VA and one urban safety net hospitals. Clinicians noted time constraints, competing demands, lack of access to decision aids and patient characteristics as barriers to shared decision making and varied in the content of information on lung cancer screening that they discussed with patients [11]. A qualitative study among 10 primary care providers in New Mexico concluded that not all participants were aware of the NLST results or USPSTF lung cancer screening guidelines, thus, few physicians felt knowledgeable enough to recommend lung cancer screening to their patients. Of those physicians who were aware of the new recommendations, many were skeptical about the efficacy of that screening test and had concerns regarding financial and geographic barriers for rural, underinsured patient populations [6].

Lewis et al. (2015) analyzed attitudes and beliefs among 212 primary care physicians based at a single academic medical center, concluding there was both low knowledge and low utilization of appropriate lung cancer screening [8]. A study among Veteran's Affairs Medical Center-based pulmonologists $(\mathrm{n}=286)$ concluded that, while respondents were familiar with national lung cancer screening guidelines, pulmonologists had varied perceptions resulting in physicians both over screening and under screening patients at risk for lung cancer [7]. Ersek et al. (2016) reported that while many family physicians $(n=101)$ were discussing LDCT with highrisk patients, this conversation occurred inconsistently and actual patient referral for LDCT remained low [12]. Carter Harris et al. (2016) compared patient-provider discussions about lung cancer screening in 2012 and in 2014, corresponding to time periods before and after release of the USPSTF guidelines and reported that patient-provider discussions regarding lung cancer screening were paradoxically more prevalent before release of lung cancer screening guidelines (17\% in 2012 and $10 \%$ in 2014), however the proportion of patient-provider discussions on this topic remained low in both time periods [13].

The purpose of this formative study was to understand attitudes and beliefs among primary care clinicians regarding LDCT lung cancer screening as well as to assess gaps in knowledge to identify opportunities for reinforcing personalized lung cancer screening that is accessible and evidence-based.

\section{Methods}

\subsection{Study Setting and Sample}

We conducted semi-structured interviews with primary care clinicians (physicians, nurse practitioners, and physician assistants) working in both Internal and Family Medicine specialties from across an 8 county region of western New York State, which includes the cities of Buffalo and Niagara Falls. By using a purposeful sampling approach, recruitment letters and e-mails were sent out to clinicians across the region to identify participants with subsequent enrichment using snow ball sampling to ensure adequate saturation of data collected. The study was approved by the cancer center's Institutional Review Board. Study staff obtained consent by reviewing the consent form with each clinician prior to proceeding with the interview.

\subsection{Data Collection}

The interview guide included a mix of 20 open-ended and closed-ended items focused on exploring domains of knowledge, attitudes and beliefs (11 items), barriers (1 item), and clinician experiences (6 items) regarding LDCT lung cancer screening. Additionally, we asked clinicians about potential opportunities for research and educational needs they perceived as important on the topic of lung cancer early detection.

Interviews were conducted during a period of several weeks in 2015 and 2016. The digitally audio-recorded interviews lasted about 30 minutes and were transcribed. Participants were offered the option of completing the interview over the phone $(n=32)$ or in person $(n=6)$. Clinicians received a $\$ 75$ gift card for participating.

\subsection{Analyses}

\subsubsection{Quantitative Analysis}

The main dependent variables included: 1) self-reported knowledge of lung cancer early detection; and 2) approaches to lung cancer early detection. Quantitative data was entered into an Excel database to be analyzed using SPSS (Version 21.0, SPSS Inc., Chicago, IL) and was summarized using descriptive statistics. No individual identifiers were collected from any respondents.

\subsubsection{Qualitative Analysis}

We used an immersion crystallization approach that involved a systematic and iterative process for reviewing interview transcripts to identify important themes [14]. All open ended items were included in the qualitative analysis and entered into NVivo 10 qualitative software (QSR International) for data management and review. Two members of the research team independently reviewed a subset of the transcripts to identify preliminary findings. The lead qualitative analyst (EMR) created a working list of topics to summarize content from the open-ended responses and create an initial coding structure (e.g. compilation of emerging codes and code descriptions) that was continually 
revised until consensus was reached among team members. The interview question guide topics were used as a framework for examining the data to develop the coding structure. Analyzing the data in this manner enhanced our ability to manage the data and improved the consistency with which we examined the interview guide topics. Each coder created a table that included the interview questions and a summary of participant responses for each item. The primary and secondary analyst/coder compared their individual analyses in order to verify agreement and detect any differences between coding. We identified patterns by analyzing codes associated with interview guide topics. Response patterns include codes that we applied to more than one participant interview when discussing a particular interview guide topic. Examination of the response patterns across the data led to the emergence and identification of themes. After reviewing these findings, the most relevant themes among the responses were identified and agreed upon by the co-authors, and illustrative quotes were chosen to represent those themes.

\section{Results}

\subsection{Quantitative}

\subsubsection{Participants}

A total of 38 clinicians engaged in medical practice throughout Western New York State were interviewed for this study. Most clinicians specialized in family medicine $(86.8 \%)$ and were physicians $(86.8 \%)$. The majority of respondents were white $(81.6 \%)$, female $(55.6 \%),<50$ years of age $(63.1 \%)$, completed their residency training $>15$ years prior $(55.3 \%)$ and reported working in single specialty $(57.9 \%)$ or multispecialty groups $(39.5 \%) ; 76 \%$ reported spending most of their professional time involved in direct patient care.

\subsubsection{Knowledge of and Experiences with Lung Cancer Screening Tests}

Table 1 summarizes how participants characterized the effectiveness, using a Likert scale, of sputum cytology, chest radiographs (CXR), and LDCT scans as lung cancer screening tests. Sputum cytology testing was characterized as either "minimally effective" (21.1\%) or "not effective" (34.2\%), although $28.4 \%$ felt that sputum cytology was "very effective" or" moderately effective", and $26.3 \%$ selected "don't know". CXR was generally described as "minimally effective" (42.1\%) or" not effective" (34.2\%), although $18.4 \%$ noted CXR to be "very effective" or "moderately effective". Low dose chest CT (LDCT) was generally rated as "very effective" (50.0\%), "moderately effective" (42.1\%) or "minimally effective" (7.9\%).

Sputum cytology for lung cancer early detection was recommended "sometimes" by $5.3 \%(\mathrm{n}=2)$ and "rarely" by $2.6 \%(\mathrm{n}=1)$. CXR screening was recommended "frequently" by $5.3 \%(\mathrm{n}=2)$, "often" by $7.9 \%(\mathrm{n}=3)$, "sometimes" by $18.4 \%(n=7)$ and "rarely" by $15.8 \%(n=6)$. Low dose CT (LDCT) chest screening was recommended "frequently" by $26.3 \%(\mathrm{n}=10)$, “often" by $21.1 \%(\mathrm{n}=8)$, "sometimes" by $23.7 \%(\mathrm{n}=9)$ and "rarely" by $23.7 \%(\mathrm{n}=9) ; 5.3 \%(\mathrm{n}=2)$ reported "never" recommending LDCT chest. [see table 1].

Table 1. Clinician opinions regarding the effectiveness and use of selected tests for lung cancer early detection, $n=38$.

\begin{tabular}{|c|c|c|c|c|c|c|}
\hline Rating of "tests" for lung cancer early detection: & & Very effective & $\begin{array}{l}\text { Moderately } \\
\text { effective }\end{array}$ & $\begin{array}{l}\text { Minimally } \\
\text { effective }\end{array}$ & Not effective & don't know \\
\hline \multirow{2}{*}{ Sputum cytology } & $\#$ & 4 & 3 & 8 & 13 & 10 \\
\hline & $\%$ & $10.5 \%$ & $7.9 \%$ & $21.1 \%$ & $34.2 \%$ & $26.3 \%$ \\
\hline \multirow{2}{*}{ Chest x-ray } & $\#$ & 1 & 6 & 16 & 13 & 2 \\
\hline & $\%$ & $2.6 \%$ & $15.8 \%$ & $42.1 \%$ & $34.2 \%$ & $5.3 \%$ \\
\hline \multirow{2}{*}{ Low dose chest CT } & $\#$ & 19 & 16 & 3 & & \\
\hline & $\%$ & $50.0 \%$ & $42.1 \%$ & $7.9 \%$ & & \\
\hline \multirow{2}{*}{ Sputum cytology } & $\#$ & & & 2 & 1 & 35 \\
\hline & $\%$ & & & $5.3 \%$ & $2.6 \%$ & $92.1 \%$ \\
\hline \multirow{2}{*}{ Chest x-ray } & $\#$ & 2 & 3 & 7 & 6 & 20 \\
\hline & $\%$ & $5.3 \%$ & $7.9 \%$ & $18.4 \%$ & $15.8 \%$ & $52.6 \%$ \\
\hline \multirow{2}{*}{ Low chest CT } & $\#$ & 10 & 8 & 9 & 9 & 2 \\
\hline & $\%$ & $26.3 \%$ & $21.1 \%$ & $23.7 \%$ & $23.7 \%$ & $5.3 \%$ \\
\hline
\end{tabular}

\subsubsection{Awareness of Lung Cancer Screening Recommendations}

As shown in Table 2, 73.7\% of respondents correctly reported that the USPSTF recommends LDCT for lung cancer early detection. About one-half of respondents indicated that the National Cancer Institute and the American Cancer Society recommended lung cancer screening with LDCT. Although the American Academy of Family Physicians (AAFP) has stated that the data on lung cancer screening with chest CT is insufficient, $55.3 \%$ of respondents incorrectly noted that the AAFP recommend lung cancer screening. Recognition of recommendations/coverage for LDCT lung cancer screening was less common for organizations such as the Centers for Medicare and Medicaid Services and the American College of Physicians (range: $23.7 \%$ to $47.4 \%$ ). 
Alvie Ahsan et al: : Examining Lung Cancer Screening Behaviors in the

Primary Care Setting: A Mixed Methods Approach

Table 2. Clinician responses regarding which of the following groups/agencies recommend lung cancer early detection using chest CT scans, $n=38$.

\begin{tabular}{|c|c|c|c|c|c|c|}
\hline \multirow{2}{*}{$\begin{array}{l}\text { Is lung cancer early detection using chest } \mathrm{CT} \\
\text { scans recommended by the........? }\end{array}$} & \multicolumn{2}{|l|}{ yes } & \multicolumn{2}{|c|}{ no } & \multicolumn{2}{|c|}{ don't know } \\
\hline & \# & $\%$ & \# & $\%$ & $\#$ & $\%$ \\
\hline National Cancer Institute & 20 & $52.6 \%$ & & & 18 & $47.4 \%$ \\
\hline American Cancer Society & 22 & $57.9 \%$ & 1 & $2.6 \%$ & 15 & $39.5 \%$ \\
\hline American Academy of Family Physicians & 21 & $55.3 \%$ & 5 & $13.2 \%$ & 12 & $31.2 \%$ \\
\hline American College of Physicians & 9 & $23.7 \%$ & 9 & $5.3 \%$ & 27 & $71.1 \%$ \\
\hline US Preventive Services Task Force & 28 & $73.7 \%$ & 6 & $15.8 \%$ & 4 & $10.5 \%$ \\
\hline Medicaid & 13 & $34.2 \%$ & 9 & $23.7 \%$ & 16 & $42.1 \%$ \\
\hline Medicare & 18 & $47.4 \%$ & 7 & $18.4 \%$ & 13 & $34.2 \%$ \\
\hline
\end{tabular}

All organizations listed except the American Academy of Family Physicians (AAFP) endorse (or provide coverage for) lung cancer screening in patients meeting eligibility criteria; the AAFP concluded that data was insufficient to recommend for or against LDCT for lung cancer screening (see text for further detail). \#, number; \%, percent.

\subsubsection{Perceived Barriers to Chest CT Lung Cancer Screening}

Perceived barriers to chest CT screening are summarized in Table 3. A majority of respondents identified limited awareness of lung cancer screening among patients $(55.3 \%)$, cost $(50 \%)$, and lack of insurance coverage $(71.1 \%)$ as major barriers. Issues such as patient anxiety $(55.3 \%)$, concern for radiation exposure $(73.7 \%)$, embarrassment about smoking $(50.0 \%)$, demands on clinician time $(63.2 \%)$, potential harms from unnecessary diagnostic procedures $(55.3 \%)$, and lower perceived usefulness were noted as minor barriers.

Table 3. Clinician opinions regarding reported barriers to Chest CT Screening for lung cancer early detection, $n=38$.

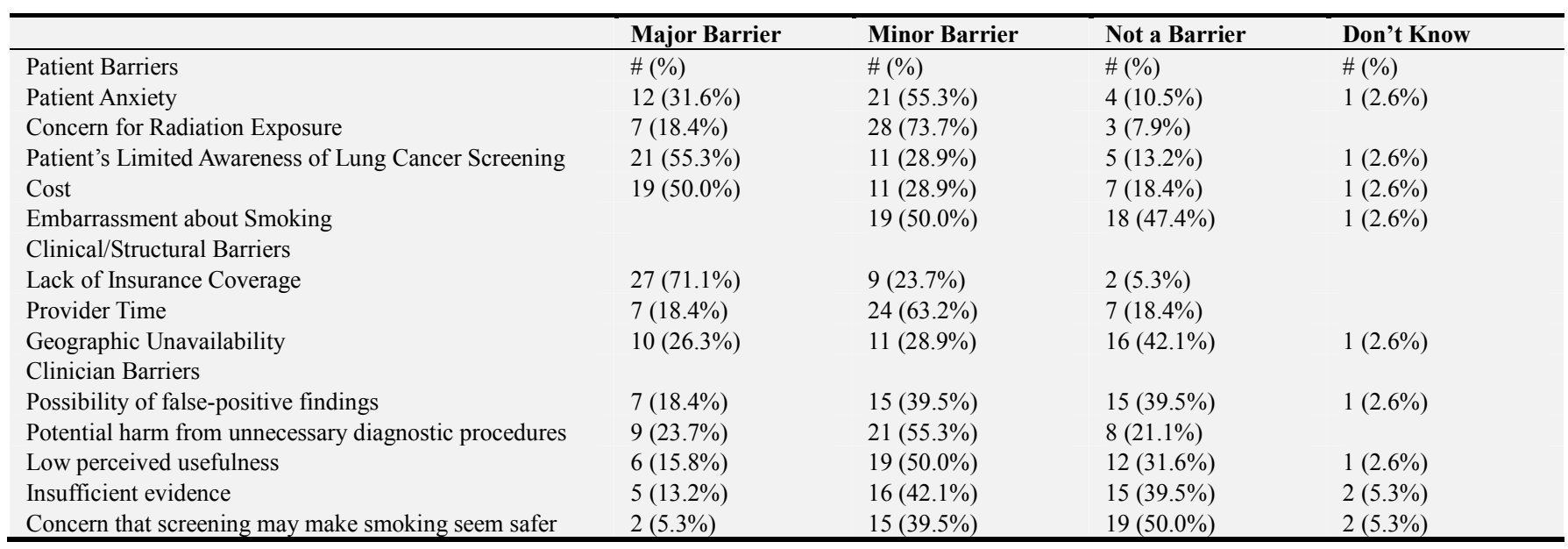

\#, number; \%, percent.

\subsubsection{Continuing Education Needs}

Most clinicians (84.2\%) endorsed a need for additional education on lung cancer screening recommendations. Preferred formats for the educational programs included onsite lectures $(52.6 \%)$, online lectures $(36.8 \%)$ or multidisciplinary conferences $(36.8 \%)$; preferred program duration was variable ( $<30$ minutes, $18.4 \%$; 31-60 minutes, $44.7 \%$; $>60$ minutes, $21.1 \%)$.

\subsection{Qualitative}

\subsubsection{Clinician Perceptions of Screening Results Regarding False Positive and False Negative Findings}

Interview participants were asked the following questions regarding their level of concern when receiving various types of results from low-dose chest computed tomography (CT) scans: "How concerned are you about false positive results from low-dose chest CT scans?" and, "How concerned are you regarding false negative results (missed cancers) from low dose chest CT scans?" Each of the questions above was followed up by a probe for whether these types of results are common/uncommon, and whether or not this was a major issue.

Nearly one-half of the participants stated that they were not very concerned about false positive test results from screening LDCT scans. Participants acknowledged that LDCT was a screening test and, like any other screening test, it would have some false positive results (Table 4, quote 1). In addition to being minimally concerned about these false positive results, some participants reported that false positive test results were an uncommon occurrence on LDCT. A smaller number of participants were very concerned about false positive test results and how it would affect their practice (Table 4, quote 2). Regardless of participants' level of concern, many clinicians felt more comfortable referring 
to a specialist to assist with patient evaluation and decisionmaking (Table 4, quote 3). A few participants claimed they did not have enough experience with the test results and thus, were not that concerned with these results.

The majority of participants were not concerned with the possibility of false negative test results and did not think false negatives were a common occurrence with LDCT. Clinicians frequently recognized that both false positive and false negative results do occur with any screening test, not just LDCT. Some believed it was best to just screen the patient. (Table 4, quote 4). Clinicians also discussed their limited experience with lung cancer screening in terms of reviewing test results and others expressed low levels of concern about false negative results due to lack of experience with LDCT.

Table 4. Selected quotes by clinicians to open-ended items on lung cancer screening, organized by theme.

\begin{tabular}{|c|c|}
\hline Theme & Clinician Quotes \\
\hline $\begin{array}{l}\text { Provider perceptions of } \\
\text { screening results } \\
\text { regarding false positive } \\
\text { and false negative } \\
\text { findings. }\end{array}$ & $\begin{array}{l}\text { 1. "It's a concern, but not a huge concern. Any screening test you have concern about false positive results." } \\
\text { 2. "Very concerned... it's going to require more follow-up investigation, more work on my part. It also increases the risk of me } \\
\text { missing the follow-up ...they have abnormal, mildly abnormal [CT], they need to repeat in } 6 \text { months...if they forget to go, then } \\
\text { it's my fault they get lung cancer later in life... Nothing is the patient's fault, it's always the doctor's fault." } \\
\text { 3. "I am concerned that there will be findings that do not represent true cancer. I know there are currently established guidelines } \\
\text { for following up pulmonary nodules based on size, shape and other characteristics. I would rely on the radiologist to provide } \\
\text { guidance as to the appropriate follow-up for nodules." } \\
\text { 4. "I think it's a relatively uncommon issue. Then again, no test is perfect and certain risk and benefits analyses show that it's } \\
\text { better to have the test done." }\end{array}$ \\
\hline $\begin{array}{l}\text { Clinician perceptions } \\
\text { on the clinical and } \\
\text { public health } \\
\text { significance of lung } \\
\text { cancer screening. }\end{array}$ & $\begin{array}{l}\text { 5. " } 30 \text { pack year history, or less than } 15 \text { years ago since their last cigarette." } \\
\text { 6. " } 30 \text { pack years or more smoking history, patients who, in addition, I would have a higher index of suspicion in patients who } \\
\text { have had a previous cancer of any sort, patients who have had a childhood cancer with chest radiation, family history, maybe some } \\
\text { complex occupational exposure, like what we talked about before-factory, truck drivers, railroad operators. And likely my index } \\
\text { of suspicion would also go up in people who have trouble maintaining their body weight." } \\
\text { 7. "Only recommend/order for those who ask for it. No pattern. Not convinced." } \\
\text { 8. "I think [these findings] are very significant. And I think it's overdue...In my practice life, there were many instances where I } \\
\text { may have been suspicious and wanted to order a CT scan of the chest and it was denied by various } 3^{\text {rd }} \text { party payers. ...the patients } \\
\text { a few years later developed lung cancer..." } \\
\text { 9. "LDCT is a good choice if you have smoking history. Decreasing chances of not finding cancer and can find cancer at early } \\
\text { stage. Decreasing health costs in the long run." } \\
\text { 10. "That's pretty significant...that means, you know, saving three lives per one thousand which is, which is great." } \\
\text { 11. "Small number of lives saved, wonder if it is cost effective and [worth] the harm from the CT scan." } \\
\text { 12. "...more clarity on what to do and what people pay for what we order." } \\
\text { 13. "Well, I'm concerned about the lower age limit: } 55 . . \text { it seems that the lower age limit should be put down to age } 45, \text { because } \\
\text { there are a lot of patients who are between age } 45 \text { and } 55 \text {, who smoked more than } 30 \text { years and they're not eligible to get } \\
\text { screening now." } \\
\text { 14. "Higher rate of preventing death vs. screening, better data for long term screening before I do it." } \\
\text { 15. "[We] may know the tip of iceberg. Especially those of us in primary care [who] manage smokers, smoking cessation is a } \\
\text { difficult problem to manage. We all need education-as you ask these questions, I'm realizing the gaps in my knowledge base." }\end{array}$ \\
\hline
\end{tabular}

LDCT, low dose computerized tomography of the chest.

\subsubsection{Identifying Patients for LDCT Screening}

The majority of providers agreed that smoking status was a key component in identifying patients for lung cancer screening and referenced following published guidelines. (Table 4, quote 5). Smoking history was the most common factor that clinicians used to refer patients for lung cancer early detection. Family history, prior history of cancer, and occupational exposures were the second most frequently reported factors considered when making patient referrals for lung cancer screening (Table 4, quote 6). Additionally, a small number of clinicians discussed additional factors or characteristics which they considered in making referrals for lung cancer screening that included sex and a patient's overall health status. For example, one clinician described recommending screening for primarily male patients. A patient's health status was also a factor providers considered in referring patients for lung cancer screening, with some contrasting viewpoints in terms of whether good health status or poor health status would better indicate who should complete screening. Finally, there were a couple of respondents who described being generally less enthusiastic about referring any patients for lung cancer screening (Table 4 , quote 7).

\subsubsection{Clinician Perceptions Regarding the Clinical and Public Health Significance of Lung Cancer Screening}

Participants were provided with the following statement: The National Lung Screening trial reported that use of lowdose chest CT scans, among high-risk smokers resulted in a $15-20 \%$ reduction in lung cancer deaths relative to screening with chest $\mathrm{x}$-rays. This reduction in the lung cancer death rate is equivalent to three fewer deaths per thousand people screened over the 6.5 years of follow-up (17.6 lung cancer deaths/1000 versus 20.7 lung cancer deaths/1000). Based on this information, do you have any thoughts or comments regarding the clinical and public health significance of these findings?

Many clinicians believed that these findings were significant and had important implications for their own practice of medicine (Table 4, quote 8 ). Similarly, another 
clinician discussed the significance of using LDCT as a screening tool for certain patient populations, and discussed the implications of these findings in the long term (Table 4, quote 9). Others expressed contrasting views on the significance of the number of lives saved using LDCT as a screening tool (Table 4, quote 10). However several respondents disagreed, and thought that the benefit of saving so few lives did not outweigh the costs associated with screening (Table 4, quote 11). Additionally, many respondents questioned what third party payers were covering it and what national organizations were supporting the guidelines (Table 4, quote 12). Clinicians also identified unanswered questions regarding the age cut offs the NLST and other guidelines proposed (Table 4, quote 13). A few respondents were concerned that more cancers were being picked up without a significant improvement in survival time. Multiple respondents requested that they would need to see rates of long term effectiveness of LDCT to understand whether the scans were actually prolonging life in addition to picking up more cancer cases (Table 4, quote 14).

Lastly, when asked how important it is to offer smoking cessation services to participants in a lung cancer early detection program, the majority of clinicians $(n=36 / 38)$ agreed that it was very important to offer smoking cessation services to these patients. Clinicians acknowledged, however, that smoking cessation is a very difficult issue to address (Table 4, quote 15).

\section{Discussion}

Based upon the USPSTF guidelines for lung cancer screening (grade B), approximately 10.5 million people in the United States would be eligible for lung cancer screening with the potential to prevent 18,000 lung cancer deaths [15]. We observed that nearly $82 \%$ of survey respondents in this study noted that recommendations from the USPSTF were relevant to their medical practice. The USPSTF also reinforced that LDCT screening recommendations should be used in addition to, rather than as a replacement for, smoking cessation interventions.[15] A grade $\mathrm{B}$ recommendation means that the USPSTF recommends the service based on a high certainty that the net benefit is moderate, or a moderate certainty that the net benefit is moderate to substantial; clinicians should offer this service [16].

It was evident that nearly all clinicians in our study recognized the importance of offering smoking cessation as an integral part of lung cancer early detection, in addition to recommending LDCT for early detections. Clinicians recognized challenges in implementing smoking cessation services and acknowledged that this was not actually happening in their clinical practice. It is important to recognize that smoking cessation is a difficult issue to address in medical settings. While the PHS tobacco dependence guidelines [17] recommend that all smokers receive at least a brief intervention as a standard of care, few smokers appear to have received cessation support as part of routine medical care $[10,18]$. Electronic medical systems have the potential to assure that all smokers received assistance with smoking and that guideline eligible patients (age 55-79, 30+ pack-year history, current smokers or quit $<15$ years) be referred for LDCT screening.

While several other national organizations including the American Cancer Society, American Association for Thoracic Surgery, American College of Chest Physicians and the National Comprehensive Cancer Network provided similar endorsements of lung cancer screening [19-22], the American Academy of Family Physicians concluded that the data was insufficient to recommend for or against LDCT screening for lung cancer [23]. By early 2015, the Centers for Medicare and Medicaid Services and private insurers started offering coverage for LDCT lung cancer screening as an additional preventive service benefit based on patient eligibility criteria that follow the recommendations released by the USPSTF and other organizations [24].

This study found that the majority of respondents were generally knowledgeable about the screening guidelines for LDCT but expressed uncertainty about age limits and lacked confidence regarding the effectiveness of this screening tool for the early detection of lung cancer. Additionally, not all clinicians who participated in interviews reported systematic procedures for referring eligible patients for screening. Findings from this study also reveal that a subset of clinicians are continuing to incorrectly recommend chest radiographs (and sputum cytology) for lung cancer early detection, which is not a screening practice recommended by any national organization, however, this observation is similar to results reported in previous studies [4, 6, 8, 9, 13]. Overall, these findings emphasize the need to continue to promote educational programs on the topic of LDCT lung cancer screening especially among those clinicians who are most likely to refer patients for screening (e.g., primary care). Clinicians in this study expressed a preference of an on-site lecture format continuing medical education program lasting between 31 and 60 minutes.

When responding to a closed-ended survey item, most clinicians identified false-positive results as a "minor barrier" to implementing LDCT lung cancer screening and in responses to an open-ended survey item few noted concerns regarding the rate of false positives. Findings from the NLST showed that of the total CT scans performed, $24.2 \%$ were "positive", however, $23.3 \%$ turned out to be false positive, resulting in a false positive rate of approximately $96.4 \%$ [5]. Some respondents to our survey claimed they did not have enough experience to evaluate the consequences of the high false positive rate; however, almost one-half of the respondents had low concern about false positives and considered this result as a common characteristic associated with all screening tests. In previous studies the majority of clinicians expressed concern about the potential harm from the high rate of false positive findings associated with $\operatorname{LDCT}[19,20]$. Also, similar to a prior report, clinicians who were concerned about false positives in this present study planned to refer positive LDCT results to a specialist for 
further evaluation, instead of handling on their own [20].

A strength and unique contribution of this study were open-ended questions to respondents to examine the topic of referral practices and what characteristics clinicians identified as relevant when referring patients for LDCT. Only one other study attempted to address this topic by providing pulmonologists with vignettes of patients who fell within or outside of guidelines [7]. Despite the USPSTF providing recommendations for the use of LDCT based primarily on smoking history and age, in this study clinicians reported recommending patients for LDCT based on other factors that may have less evidence to support patient referral (e.g., sex, family history, past history of malignancy, occupational exposures). Guidelines from the National Comprehensive Cancer Network (NCCN) do note occupational exposures and family history as pertinent characteristics of patients who should be screened with LDCT, however the evidence supporting those recommendations is less robust, unlike the evidence supporting the USPSTF guidelines, which are more widely accepted $[5,25]$. However, inconsistency in lung cancer screening guidelines/recommendations from national organizations likely contributed to how clinicians responded in this study and/or may also be related to clinicians' challenges to stay up to date with screening guidelines and recommendations as reported in another study [6].

Many clinicians in this study were left with unanswered questions after hearing the results of the NLST. The significance of the high number of screenings that were required to prevent a single lung cancer death, the distinct age cut offs, as well as the significance of the number of years of life gained after detecting a positive screen were topics about which most clinicians had remaining questions. These findings were very similar to another study that also showed that clinicians were skeptical of evidence interpreted from the NLST [5]. Hoffman et al. also found that the number of screens that needed to be done to prevent one lung cancer death was high, and clinicians also wanted to see more evidence on the use of LDCT screening on different populations in order to fully accept these guidelines and recommendations [6]. Data from NLST indicate a number needed to screen (NNS) to prevent 1 lung cancer death of 333 which compares to NNS of 1410-1254 for prostate screening over 9 years [26], 781 for breast screening over 8 years, and 1250 for colorectal screening over 5 years to prevent 1 cancer-specific death [27].

The somewhat narrow range of clinical professionals and specialties recruited from within this defined geographic region, and issues related to participant recruitment, may limit the generalizability of our findings. However, our mixed methods approach contributes to a more robust understanding of how primary care clinicians perceive the efficacy and use of lung cancer screening is crucial to promoting LDCT lung cancer screening and to achieving potential reductions of lung cancer mortality.

\section{Conclusion}

Our findings suggest that primary care clinicians endorse the effectiveness of LDCT for lung cancer early detection but are less frequently recommending LDCT to their patients. Some respondents reported using additional patient characteristics, including sex, family history, past medical history, and occupational exposures, to guide lung cancer screening beyond the recommended eligibility criteria which supports a need for professional education to address knowledge gaps. In addition, the development and dissemination of decision support tools may also help to enhance and promote use of LDCT lung cancer screening.

\section{Acknowledgements}

This study was supported in part by Roswell Park Comprehensive Cancer Center and National Cancer Institute (NCI) grants 3P30CA01605 and U54CA153598.

\section{Conflict of Interest}

The authors declare that they have no conflicts of interest.

\section{References}

[1] Centers for Disease Control and Prevention (2017a). United States cancer statistics: 1999-2014 incidence and mortality web-based report. 2017; www.cdc.gov/cancer/uscs. Accessed February 14, 2019.

[2] Centers for Disease Control and Prevention (2017b). Smoking and cancer. 2017; https://www.cdc.gov/tobacco/campaign/tips/diseases/cancer. html\#how-related. Accessed February 14, 2019.

[3] Noone AM, Howlader N, Krapcho M, Miller D, Brest A, Yu M, Ruhl J, Tatalovich Z, Mariotto A, Lewis DR, Chen HS, Feuer EJ, Cronin KA (eds). SEER Cancer Statistics Review, 1975-2015, National Cancer Institute. Bethesda, MD, https://seer.cancer.gov/csr/1975 2015/, based on November 2017 SEER data submission, posted to the SEER web site, April 2018; https://seer.cancer.gov/statfacts/html/lungb.html. Accessed February 14, 2019.

[4] Moyer VA. Screening for lung cancer: US Preventive Services Task Force recommendation statement. Annals of internal medicine. 2014; 160(5):330-338.

[5] National Lung Screening Trial research Team (2011). Reduced lung-cancer mortality with low-dose computed tomographic screening. New England Journal of Medicine. 2011;365(5):395-409.

[6] Hoffman RM, Sussman AL, Getrich CM, et al. Peer Reviewed: Attitudes and Beliefs of Primary Care Providers in New Mexico About Lung Cancer Screening Using LowDose Computed Tomography. Preventing chronic disease. $2015 ; 12$.

[7] Iaccarino JM, Clark J, Bolton R, et al. A national survey of pulmonologists' views on low-dose computed tomography screening for lung cancer. Annals of the American Thoracic Society. 2015; 12(11):1667-1675. 
[8] Lewis JA, Petty WJ, Tooze JA, et al. Low-dose CT lung cancer screening practices and attitudes among primary care providers at an academic medical center. Cancer Epidemiology and Prevention Biomarkers. 2015; 24(4):664670.

[9] Smetana GW, Boiselle PM, Schwartzstein RM. Screening for lung cancer with low-dose computed tomography: grand rounds discussion from the Beth Israel Deaconess Medical Center. Annals of internal medicine. 2015; 162(8):577-582.

[10] Kanodra NM, Pope C, Halbert CH, Silvestri GA, Rice LJ, Tanner NT. Primary Care Provider and Patient Perspectives on Lung Cancer Screening. A Qualitative Study. Ann Am Thorac Soc. 2016; 13(11):1977-1982.

[11] Wiener RS, Koppelman E, Bolton R, et al. Patient and Clinician Perspectives on Shared Decision-making in Early Adopting Lung Cancer Screening Programs: a Qualitative Study. J Gen Intern Med. 2018; 33(7):1035-1042.

[12] Ersek JL, Eberth JM, McDonnell KK, et al. Knowledge of, attitudes toward, and use of low-dose computed tomography for lung cancer screening among family physicians. Cancer. 2016; 122(15):2324-2331.

[13] Carter-Harris L, Tan AS, Salloum RG, Young-Wolff KC. Patient-provider discussions about lung cancer screening preand post-guidelines: Health Information National Trends Survey (HINTS). Patient education and counseling. 2016; 99(11):1772-1777.

[14] Borkan J. Immersion/crystallization In: Crabtree BM, WL, ed. Doing qualitative research. Thousand Oaks, CA: Sage; 1999.

[15] US Preventive Task Force (2013a). Final recommendation statement, Lung cancer: Screening. 2013; https://www.uspreventiveservicestaskforce.org/Page/Documen $\mathrm{t} /$ RecommendationStatementFinal/lung-cancer-screening. Accessed February 14, 2019.

[16] US Preventive Task Force (2013b); https://www.uspreventiveservicestaskforce.org/Page/Name/gra de-definitions. Accessed April 16, 2018.

[17] Fiore MC, Jaen CR, Baker T, et al. Treating tobacco use and dependence: 2008 update. Rockville, MD: US Department of Health and Human Services. 2008.

[18] Mahoney MC, Twarozek AM, Saad-Harfouche F, et al.
Assessing the delivery of cessation services to smokers in urban, safety-net clinics. Journal of community health. 2014; 39(5):879-885.

[19] Detterbeck FC, Mazzone PJ, Naidich DP, Bach PB. Screening for lung cancer: diagnosis and management of lung cancer: American College of Chest Physicians evidence-based clinical practice guidelines. Chest. 2013; 143(5): e78S-e92S.

[20] Jaklitsch MT, Jacobson FL, Austin JH, et al. The American Association for Thoracic Surgery guidelines for lung cancer screening using low-dose computed tomography scans for lung cancer survivors and other high-risk groups. The Journal of thoracic and cardiovascular surgery. 2012;144(1):33-38.

[21] National Comprehensive Cancer Network (2018). NCCN Lung cancer screening, version 2.2019 - August 27, 2018; https://www.nccn.org/professionals/physician_gls/pdf/lung_sc reening.pdf. Accessed February 14, 2019.

[22] Wender R, Fontham ET, Barrera E, et al. American Cancer Society lung cancer screening guidelines. CA: a cancer journal for clinicians. 2013; 63(2):106-117.

[23] American Academy of Family Physicians (2019). Clinical preventive service recommendation. 2018; https://www.aafp.org/patient-care/clinicalrecommendations/all/lung-cancer.html. Accessed February 14, 2019.

[24] Centers for Disease Control and Prevention (2017a). Decision memo for screening for lung cancer low dose domputed tomotgraphy (LDCT) (CAG-00439N). 2018; https://www.cms.gov/medicare-coverage-database/details/ncadecision-memo.aspx?NCAId=274. Accessed February 14, 2019.

[25] Poonacha TK, Go RS. Level of scientific evidence underlying recommendations arising from the National Comprehensive Cancer Network clinical practice guidelines. Journal of Clinical Oncology. 2010; 29(2):186-191.

[26] Loeb S, Vonesh EF, Metter EJ, Carter HB, Gann PH, Catalona WJ. What is the true number needed to screen and treat to save a life with prostate-specific antigen testing? Journal of clinical oncology: official journal of the American Society of Clinical Oncology. 2011; 29(4):464-467.

[27] Richardson A. Screening and the number needed to treat. Journal of medical screening. 2001; 8(3):125-127. 